\title{
IMPORTÂNCIA DA MICROBIOTA INTESTINAL E SUA MODULAÇÃO PARA O EMAGRECIMENTO SAUDÁVEL
}

\author{
Ana Luiza Gandra ${ }^{1}$ \\ Bruna Gonçalves Flausino ${ }^{2}$ \\ Bárbara Xavier Barbosa Martins ${ }^{3}$ \\ Débora Cristina Rodrigues Sousa ${ }^{4}$ \\ Dóris Cristina Freitas Dolabela ${ }^{5}$ \\ Elaine Miranda Padilha Duarte ${ }^{6}$ \\ Lhorena Paula da Silva Lourenço ${ }^{7}$ \\ Shênia Miranda Ribeiro ${ }^{8}$ \\ Mirian Cristina de Oliveira ${ }^{9}$
}

RESUMO: Atualmente os probióticos vem ganhando relevância entre indivíduos e na área médica tanto por apresentar grande benefícios para nossa microbiota como no auxílio de algumas doenças. Além disso, atuais estudos mostram sua eficácia contra obesidade. A inclusão de alimentos simbióticos na alimentação humana tende a estimular o crescimento de determinados microrganismos benéficos para o hospedeiro. Para manter a microbiota equilibrada é importante uma alimentação rica em probióticos contribuindo para digestão, absorção de nutrientes fortalecendo o sistema imunológico. Este trabalho tem como objetivo realizar uma revisão de literatura sobre a importância da microbiota intestinal e sua modulação para o emagrecimento saudável e apresentar alguns probióticos que auxiliem no seu equilíbrio. Para alcançar os objetivos propostos, utilizou-se como recurso metodológico, a pesquisa bibliográfica exploratória com caráter descritivo, tendo como bases documentos extraídos de dados virtuais Scientific Electronic Library Online (Sciello), Google acadêmico e demais sites científicos, para obtenção de artigos, teses, monografias, dissertações e livros envolvidos no tema proposto.

Palavras-chave: Trato gastrointestinal. Microbiota intestinal Emagrecimento e Probióticos.

\footnotetext{
${ }^{r}$ Acadêmica do curso de graduação de Biomedicina da Faculdade Única de Ipatinga/MG;

${ }^{2}$ Acadêmica do curso de graduação de Biomedicina da FaculdaCde Única de Ipatinga/MG;

${ }^{3}$ Acadêmica do curso de graduação de Biomedicina da Faculdade Única de Ipatinga/MG;

${ }^{4}$ Acadêmica do curso de graduação de Biomedicina da Faculdade Única de Ipatinga/MG;

${ }^{5}$ Acadêmica do curso de graduação de Biomedicina da Faculdade Única de Ipatinga/MG;

${ }^{6}$ Acadêmica do curso de graduação de Biomedicina da Faculdade Única de Ipatinga/MG;

${ }^{7}$ Acadêmica do curso de graduação de Biomedicina da Faculdade Única de Ipatinga/MG;

${ }^{8}$ Acadêmica do curso de graduação de Biomedicina da Faculdade Única de Ipatinga/MG;

9 Professora de Microbiologia Básica e Microbiologia Clínica da Única de Ipatinga M.G. Email:mirianbiom@gmail.com.
} 
ABSTRACT: Currently, probiotics are gaining relevance among individuals and in the medical field, both for presenting great benefits for our microbiota and for helping with some diseases. Furthermore, current studies show its effectiveness against obesity. The inclusion of symbiotic foods in human nutrition tends to stimulate the growth of certain microorganisms that are beneficial to the host. To keep the microbiota balanced, a diet rich in probiotics is important, contributing to digestion, absorption of nutrients and strengthening the immune system. This work aims to carry out a literature review on the importance of the intestinal microbiota and its modulation for healthy weight loss and to present some probiotics that help in its balance. To achieve the proposed objectives, exploratory bibliographic research with a descriptive character was used as a methodological resource, based on documents extracted from virtual data Scientific Electronic Library Online (Sciello), academic Google and other scientific sites, to obtain articles, theses, monographs, dissertations and books involved in the proposed theme.

Keywords: Gastrointestinal tract. intestinal microbiota. weight loss and probiotics.

\section{INTRODUÇÃO}

A microbiota intestinal humana, responsável no metabolismo, convertendo alimento

em nutriente e energia, é composta de io a ıoo trilhões de bactérias, vírus e fungos, envolvendo mais de mil espécies e correspondendo a io vezes o número de células presentes no corpo humano (ANDRADE et al.,2015; OLIVEIRA; HAMMES, 2016).

Dessas bactérias, estima-se que $70 \%$ estejam no intestino grosso e delgado, coexistindo e interagindo com o objetivo de defender contra infecção de microrganismo patogênicos, realizar função metabólica, com elaboração de uma ampla variedade de nutrientes e ter finalidade de imunomodulador. (OLIVEIRA; HAMMES, 20I6).

Andrade et al. (2015) definem obesidade como "uma doença crônica que resulta no acúmulo excessivo de gordura corporal".

É considerada uma epidemia de grande impacto na saúde pública da sociedade ocidental, uma vez que é diretamente relacionada com diferentes comorbidades como "síndrome metabólica, diabetes e hipertensão". É definida e classificada pela Organização Mundial da Saúde (OMS) de acordo com o índice de massa corporal (IMC), sendo considerado obeso o indivíduo com valores iguais ou maiores a $30 \mathrm{~kg} / \mathrm{m} 2$ (OLIVEIRA; HAMMES, 2016).

Segundo Censo 2019 do IBGE, a população com sobrepeso do Brasil é de 6I,7\%, sendo que a maioria dos casos é relacionado ao fator nutricional do indivíduo. Além disso, o desequilíbrio da microbiota intestinal humana $(\mathrm{MIH})$ tem sido um forte fator de sobrepeso (LORENZ; OZORIO, 2019).

A microbiota das pessoas obesas e magras é distinta e podem serem responsáveis pelo desenvolvimento da obesidade (ANDRADE et al., 2015). 
Existe uma grande variedade de microrganismos que compõem a microbiota intestinal humana $(\mathrm{MIH})$, sendo que o desequilíbrio das mesmas pode levar o indivíduo a um sobrepeso. Presentes no trato gastrointestinal (TGI), a microbiota auxilia no processo metabólico com um desenvolvimento notoriamente grande, e o fator nutricional resulta em um sobrepeso (SALOMÃO et al, 2020).

O presente artigo tem como objetivo realizar uma revisão literária acerca da importância da microbiota intestinal e sua modulação para seu equilíbrio. Conhecendo os benefícios dos probióticos para uma regulação intestinal e melhor qualidade de vida.

\section{MATERIAL E MÉTODOS}

O trabalho foi desenvolvido a partir de uma revisão de literatura obtida por meio das ferramentas de busca Scientific Electronic Library Online (SciELO) e Google Acadêmico, com as palavraschaves em língua portuguesa: trato gastrointestinal, microbiota intestinal, disbiose, emagrecimento e probióticos.

Para o trabalho descritivo, foram selecionados artigos científicos, dissertações e teses, com publicações ocorridas a partir de 1992 até 2020 da língua vernácula e estrangeira que atendesse ao propósito da pesquisa.

Foram excluídos artigos de e monografias, que não apresenta relevância da publicação para o trabalho e para o assunto. Os artigos foram analisados e selecionados criteriosamente conforme a relevância para o estudo, e em seguida foram agrupados por assunto. Posteriormente ocorreu a discussão, construção e apresentação da revisão de literatura.

\section{DESENVOLVIMENTO}

\section{Microbiota intestinal}

A microbiota intestinal, formada no período pós-natal, é constituída por uma infinidade de bactérias, em sua maioria, anaeróbias (GUARNER, 2007; BARBOSA et al., 2010), que realizam diversas funções no corpo humano, tais como: proteção contra patógenos, absorção de nutrientes e modulação do sistema imune (MORAES et al., 2014). Desse modo, ela é formada por microbiota nativa e microbiota de transição temporária, além de ser considerada um dos ecossistemas mais complexos, o conjunto desses micro-organismos é composto por cerca de 1000 espécies de bactérias distintas. O corpo humano, aos dois anos de idade, estabiliza sua colonização podendo comparar à de um adulto (GUARNER, 2007; BARBOSA et al., 2010).

No nascimento, durante a passagem pelo canal vaginal, inicia-se a influência na formação da colonização do trato gastrointestinal (TGI) do recémnascido. Essa ocorrência tem maior predominância no parto normal, tal fato deve-se ao contato direto com a microbiota fecal da mãe. Após o período neonatal, o ambiente e a amamentação também induzem a flora microbiana, sendo influenciada pelo leite humano ou leite industrializado (PENNA; NICOLLI, 200I).

Nesse sentido, destaca-se que a microbiota intestinal é de grande importância para saúde do indivíduo, desde a fase recém-nascida até a fase adulta. $\mathrm{Na}$ sua colonização ocorrem modificações ao longo da vida, a sua instalação pode diminuir a proliferação e disseminação 
de bactérias multirresistentes. Dessa forma, contribui com favoráveis funções como por exemplo: as antibacterianas, imunomodulação e metabólicos nutricionais (BRANDT, SAMPAIO, MIUKI, 2006; WALL et al., 2009).

A microbiota entérica é composta por bactérias benéficas ou probióticas e as nocivas, sendo como exemplo de probióticas as bifidobacterias e os lactobacilos (Bacteroides spp., Bifidobacterium spp., Lactobaciluus spp). Já entre as nocivas pode-se citar a família Enterobacteriaceae e Clostridium spp. Além disso, na microbiota entérica também é encontrado a Eubacterium spp., Funsonbacterium spp, Peptostrepto spp., Ruminococcus (SANTOS, VARAVALHO, 20II).

Os probióticos são usados em medicina humana na regulação da microbiota intestinal e, ao serem administrados em quantidades adequadas, acarretam em diversos efeitos positivos para a saúde de seu hospedeiro. Dentre seus efeitos é possível destacar a diminuição da permeabilidade intestinal, a proteção contra invasores patogênicos e estimulação do sistema imunológico (HAVENAAR; HUIS IN'T VELD, 1992).

\section{Microbiota no trato gastrointestinal}

A microbiota é a composição de todos os microrganismos que habitam o tubo digestivo e as mucosas intestinais incluindo fungos, bactérias e vírus, sendo a maior parte bactérias. As bactérias mais predominantes são os membros do filo dos Bacteroidetes e Firmicutes. Elas exercem diversas funções desde a proteção de membrana, produção de vitaminas, substâncias químicas que funcionam como hormônios comunicando diretamente com cérebro através do nervo vago.

(Fiocchi e Pereira de Sousa, 2012, Lozupone et al., 2012)

No trato gastrointestinal (TGI) encontramos populações bacterianas que colonizam o ser humano (Figura I) variando a espécie de acordo com a região que se encontra. As bactérias estão em maior quantidade no intestino e no cólon e lá a grande atividade metabólica pois esses órgãos são propícios para sua proliferação, melhorando a digestão e a absorção dos nutrientes, assim como, resposta imune contribuindo para a homeostase da nossa saúde (PRAKASH et al., 20II).

(Adaptado de Porto et al., 2014).

Embora a microbiota esteja dividida em outras áreas de contato, a colonização ocorre cerca de $70 \%$ no trato gastrointestinal sendo constituídas por centenas de bactérias. Encontram-se divididas entre espécies transitórias que circulam ao longo do trato digestivo, como as leveduras e bactérias lácticas. Espécies raras, são exemplo Streptoccocus, Echerichia coli, Enterobacteriacae e espécies dominantes como Cloristridium, Bacteroides, Bifidobacterium. No entanto esses grupos definem a sua microbiota intestinal tendo relação com hábitos alimentares dieta, consumo de antibióticos, estilo de vida e idade seu desequilíbrio pode levar a alterações na regulação. (Porto, 2014) 


\section{Microbiota intestinal}

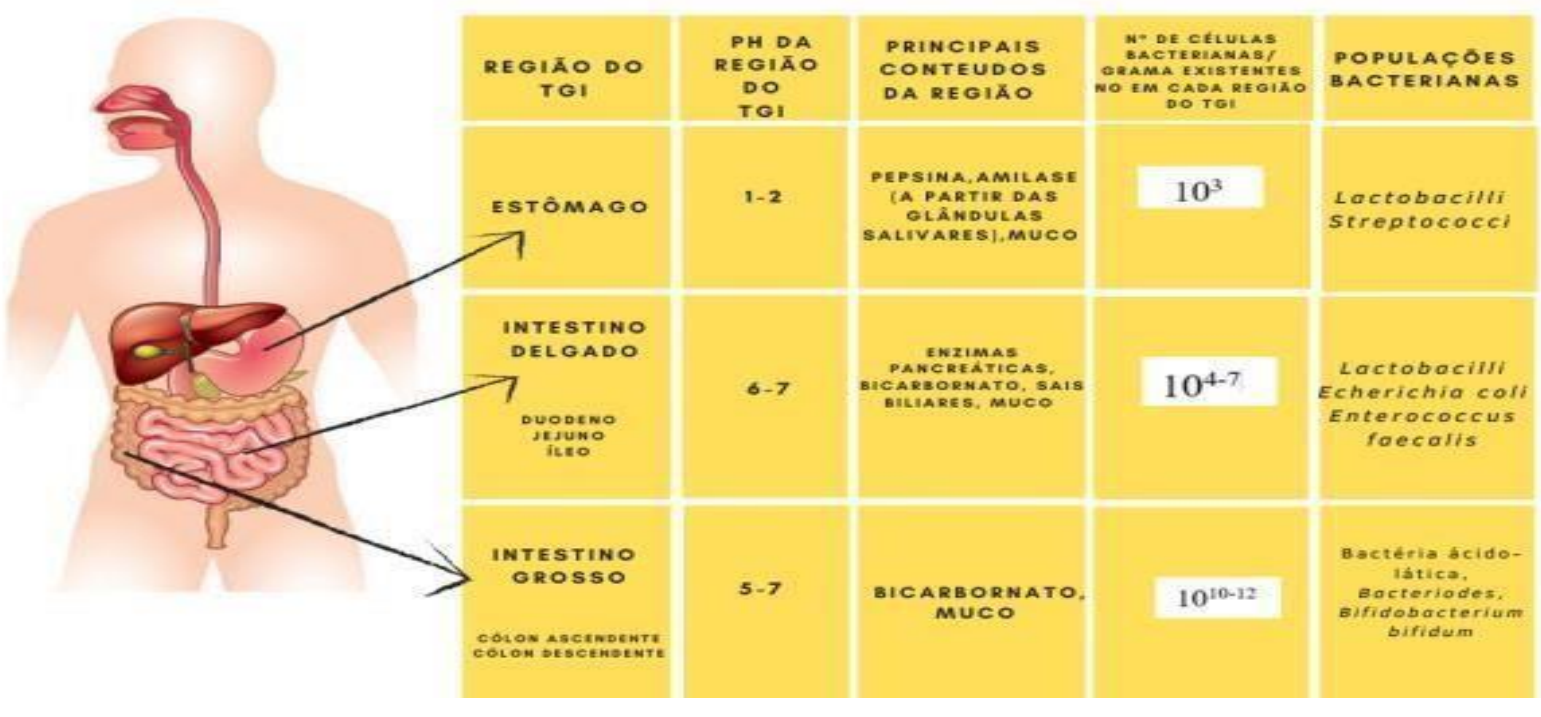

Figura 1. Espécies bacterianas na microbiota intestinal em diferentes regiões trato gastrointestinal, principais conteúdos e populações bacterianas.

\section{Microbiota e emagrecimento}

A obesidade é uma doença crônica sendo um problema de saúde pública que vem chamando a atenção pelo seu crescimento em todas as faixas etárias, seu aspecto se dá pelo excesso de gordura corporal, sendo sua etiologia o estilo de vida, má alimentação, sedentarismo, distúrbios hormonais e fatores genéticos (WANDERLEY; FERREIRA, 2010). Popularmente, a microbiota intestinal é conhecida como flora intestinal e ela é povoada por inúmeros micro-organismos como fungos, bactérias e vírus, sendo que o primeiro contato se inicia no parto, durante a passagem pelo canal do parto ou pelo contato com a pele da mãe dando início a formação da microbiota do trato gastrointestinal do recém-nascido, proporcionando o bom funcionamento do intestino, auxiliando na ativação do sistema imune, promovendo uma reposta imunológica, com boa absorção e produção de nutrientes, tais como vitamina $\mathrm{K}$ e do complexo $\mathrm{B}$. (PRAKASH et al., 2orI).

Através da microbiota intestinal humana (MIH) a obtenção de energia dos alimentos e o metabolismo lipídico e até mesmo a resposta imune pode sofrer influência na sua função endócrinas, fazendo assim a diferenciação entre indivíduo magro e gordo. Algumas alterações na colonização tem sido avaliadas para explicar a importância da MIH no indivíduo com obesidade. Segundo Salomão, Joab, Oliveira 2020 uma das variantes está relacionada a regulação de energia e a habilidade de fermentar polissacarídeos não alterado pela microbiota. Outra alteração proposta e a predisposição da MIH em reduzir a oxidação de ácidos graxos hepáticos por meio da supressão da adenosina monofosfato quinase (AMPK).

A disbiose intestinal também está presente na síndrome metabólica com menor prevalência de Bacteroidetes e aumento da prevalência de população de Firmicutes. Segundo 
Lee (200I) e Myers Jr et al.(2010) os estudos realizados em ratos com mutação no gene que codifica para a leptina demostram um aumento do apetite e uma alteração na composição do microbiota intestinal em indivíduos com predominância de bactéria Firmicutes em comparação com o filo Bacteroidetes.

No estudo realizado por Andrade et al. (2015) aponta que a microbiota intestinal de obesos pode apresentar uma peculiaridade pela indução da inflamação crônica, ou seja, a provocação da endotoxemia pela obesidade, diabetes mellitus e resistência à insulina favorece o aparecimento de processos inflamatórios, semelhantes aos presentes em pessoas com dieta com alto teor de gordura.

O estilo de vida tem grande influência na obesidade, no emagrecimento e nas doenças metabólicas. A diversidade da microbiota intestinal humana (MIH) sofrendo alteração em sua composição de forma positiva com a mudanças de hábitos alimentares podemos organizar a MIH com uma alimentação saudável e equilibrada com produtos que aumente a Bacteriodetes, com a prática de atividade física e diminuição de pães, massas, arroz e doces. Ajudando assim a restauração da microbiota intestinal a um estado de homeostase com isso melhorando as condições do organismo para ajudar a manter um peso saudável. (Salomão, Joab oliveira 2020).

Salomão et al. (2020) expõem em seu estudo que a MIH pode influenciar diretamente o metabolismo do hospedeiro, uma vez que determinadas cepas, agem com pressão seletiva sobre as demais.

Ainda sobre o desequilíbrio da MIH é coeso afirmar que de acordo com algumas pesquisas sobre este referido assunto, indivíduos com sobrepeso tendem a apresentar a MIH com microrganismos diferentes dos indivíduos que não apresentam sobrepeso. Os genes são modificados, por conseguinte, conforme os microrganismos presentes, e, por conseguinte os indivíduos ficam mais propensos ao aumento do tecido adiposo (SANTOS; FURTADO; PERES, 2019).

Hábitos diários com ingesta de alimentos industrializados podem gerar uma disbiose causando uma desregulação na saúde da microbiota, aumentando a toxicidade do intestino dando a oportunidade para colonização de bactérias patogênicas ou até oportunistas. A disbiose também pode ser induzida por outros fatores como o uso de antibióticos e laxantes de forma abusiva e sem prescrição medica que acabam alterando a quantidade de bactérias residentes e as oportunistas na colonização da microbiota (ALMEIDA et al., 2009).

\section{Importância da microbiota para o emagrecimento}

Segundo BAOTMAN et al., (2016) A microbiota intestinal (MIH) chamou atenção na última década como um elemento que afeta diretamente o estado de saúde ou doença do indivíduo, implicando na etiologia da obesidade e atualmente podemos perceber que a obesidade no Brasil vem se tornando um problema de saúde pública além disto vários indivíduos enfrentam dificuldades com o processo de emagrecimento. Estudos levantam a hipótese de que a obesidade pode estar relacionada com alterações da microbiota, a explicação se deve a desregulação do ecossistema intestinal afetando a produção e regulação de energia. (SIRTULI. J ,2017)

A microbiota intestinal e constituída já no primeiro ano de vida, a mesma é influenciada por vários fatores, como o fator idade gestacional, tipo de parto e a dieta, esses fatores têm efeitos significativos sobre a microbiota (MALOZI,20Io) e pode ser vista como 
um órgão metabólico sinergicamente ajustado à fisiologia humana envolvendo mais de mil espécies em relação de simbiose com o organismo. O qual executa funções que nosso organismo não conseguiu evoluir a ponto de desempenhá-las sozinho. Estas funções incluem a habilidade de processar componentes dietéticos indigeríveis, e função antibacteriana, pois as bactérias exercem proteção ecológica intestinal, impedindo o estabelecimento das bactérias patogênicas. Além disso possui função imunomoduladora, interagindo com as células do epitélio intestinal do hospedeiro e provocando uma resposta contínua do sistema imune. Este, por sua vez, tende a desenvolver-se e constitui importante componente do sistema imune (LAGE e BRITO,

2012).

Alguns estudos vem mostrando que a obesidade pode estar relativamente ligada a disbiose, que é um desequilíbrio entre bactérias protetoras e agressoras no intestino ou estômago onde as bactérias patogênicas tem domínio sobre bactérias benéficas, alterando assim a absorção de nutrientes no intestino, ela pode desencadear uma constipação (intestino preso) ou diarreia, ou quadros mais complexos como as alterações na barreira mucosa, aumento da absorção de substâncias tóxicas e de bactérias prejudiciais à saúde. Além de estimular sistema imunológico de maneira desorganizada, a disbiose facilita o ganho de peso, e aumento de gordura corporal devido aumento do hormônio cortisol. (Durço e Maynard, BRASÍLIA, 2018).

A maioria dessa composição bacteriana da MIH é representa por Bacterioides e Firmicutes. Wagner et al. (2018), em seu trabalho de revisão apresentaram importante estudo no qual as Bacteroidetes e as Firmicutes, constituem mais de $90 \%$ das categorias filogenéticas conhecidas e dominantes no intestino distal e que, comparando a microbiota intestinal de indivíduos magros e obesos, os indivíduos obesos apresentavam uma proporção reduzida de Bacteroidetes e níveis mais elevados de Firmicutes. Seu estudo também demonstrou que, após o tratamento realizado com dieta, a abundância relativa de Bacteroidetes aumentou enquanto a de Firmicutes diminuiu. E, ainda de acordo com o referencial teórico, o filo Firmicutes contempla mais de 200 gêneros, muitos deles com maior eficiência em extrair calorias de carboidratos que o filo bacteroidetes. Constatou-se então, que a quantidade aumentada de bacteroidetes estava relacionada com a porcentagem de perda de peso corporal e não a quantidade calórica da dieta. (LAGE e BRITO, 2012)

Deste modo o desequilíbrio da microbiota intestinal pode ser um fator importante para a obesidade. A manipulação, tanto da dieta, como por meio de fármacos, pode representar uma nova estratégia para o tratamento da obesidade e suas complicações, uma vez que a microbiota equilibrada vai ajudar na função fisiológica normal humana (PISTELLI e COSTA, 20I0). Contudo a microbiota além de ser importante na fisiopatologia humana é importante para nossa saúde em relação a possíveis doenças sendo os componentes da dieta valioso para sua formação.

\section{Probiótico}

Definidos pela OMS como micro-organismos vivos que auxiliam na saúde dos seus hospedeiros, os probióticos são identificados por suas diferentes cepas específicas (SOARES, 2019). O termo probiótico originou-se do grego, que significa "para a vida". Foi a princípio usado por Lilly e Stillwell6. São complementos alimentares baseado em microrganismos 
vivos que atingem o hospedeiro de forma benéfica, permitindo o balanço de sua microbiota intestinal. Os gêneros mais aplicados são Lactobacillus e Bifidobacterium. Os probióticos vem ganhando cada vez mais força e relevância entre indivíduos e na área medica tanto por apresentar grande benefício para nossa microbiota como no auxílio de algumas doenças. Além disso atuais estudos demonstram sua eficácia contra obesidade. (Frota, K; et al 2015)

Dentre os gêneros mais utilizados de probióticos em produtos alimentícios estão os Lactobacillus e Bifidobacterium, que a partir da atividade fermentativa geram compostos orgânicos que alteram a acidez intestinal e impossibilitam o crescimento de microorganismos patógenos (SOARES, 2019).

No Japão, em 1930, foi isolada uma espécie de Lactobacillus que é muito usada na fabricação de um leite fermentado conhecido mundialmente.

Metchnikoff analisou os benefícios dos probióticos ao relacionar o uso de leite fermentado com a resistência de campesinos búlgaros. Os efeitos das bactérias probióticas só mostram efeitos biológicos no meio intestinal se alcançarem uma quantidade mínima. Diversos autores recomendam que os produtos lácteos de $100 \mathrm{~g}$ tenha pelo menos ${ }_{10}{ }^{7} \mathrm{UFC} / \mathrm{g}$ de Lactobacillus rhamnosus para diminuir consideravelmente a incidência da diarréia dos viajantes. (Stürmer, E; et al., 2012)

Segundo GONÇALVES (2012) A maior parte dos probióticos é composta de bactérias ácidoláticas, Gram-positivas, normalmente catalase-negativas, que desenvolvem em microaerofilia. As espécies ácidoláticas dos probióticos incluem os gêneros Bifidobacterium, Enterococcus, Lactobacillus,

Lactococcus, Leuconostoc, SporoLactobacillus Pediococcus e Streptococcus; espécies não ácidoláticas, como; Bacillus cereus, Escherichia coli e Propionibacterium freudenreichii; e as leveduras Saccharomyces cerevisiae e Saccharomyces boulardii. E sua ingestão possui vários benefícios como combater e prevenir doenças intestinais, combater doenças, melhorar a digestão, combater a prisão de ventre e a diarreia, aumentar a absorção de nutrientes, como vitamina $B$, cálcio e ferro, fortalecer o sistema imunológico, por aumentar a produção de células de defesa chamadas macrófagos, impedir a propagação de bactérias ruins no intestino ajudar a digerir a lactose, prevenir problemas como obesidade, colesterol alto e hipertensão. (Fontané, L; et al., 2018)

Ao longo dos últimos anos, foram geradas informações indicando que diversos probióticos têm, além da sua atuação de regular a microbiota das mucosas e como agentes de crescimento, efeito imunomodulador. A inserção de alimentos probióticos na alimentação humana tende a impulsionar o crescimento de certos microrganismos benéficos para o hospedeiro. Houve, nos últimos anos, um considerável aumento de interesse pelos benefícios terapêuticos dos probióticos. (GONÇALVES 2012).

Para Soares (2010) a utilização dos probióticos nos produtos alimentícios é determinada a partir da resistência apresentada após a ingestão, a fim de que chegue de maneira segura ao intestino e na quantidade adequada para que a cepa possa agir.

Os probióticos são utilizados na disbiose intestinal com o intuito de prevenir e tratar o sobrepeso, sendo utilizados microrganismo para o equilíbrio $\mathrm{MIH}$, sendo utilizado nas composições dos probióticos Lactobacilluse e Bifidobacterium (NUNES; GARRIDO, 2018). Assim, podemos perceber a importância dos probióticos na saúde humana, apresentando vários benéficos e podendo ser encontrado tanto em suplemento quanto em alimentos, para 
manter a microbiota equilibrada e importante uma alimentação rica em probióticos contribuindo para digestão, absorção de nutrientes e fortalecer o sistema imunológico.

\section{CONCLUSÃO}

Com base no que foi apresentado, compreendese que a microbiota intestinal humana (MIH) tem grande importância para o ser humano no auxílio da regulação do intestino, melhorando o sistema de defesa, digestão e absorção de nutrientes evitando assim comorbidades como a síndrome metabólica, diabetes, hipertensão e até a obesidade.

\section{REFERÊNCIAS}

[1] ANDRADE, V. L. A. et al. Obesidade e microbiota intestinal microbiota. Revista Médica de Minas

Gerais, [S.L.], v. 25, n. 4, p. 583-589, 2015.

[2] FIOCCHI, C. e Pereira de Sousa, H.S. Microbiota Intestinal - Sua importância e função. Jornal Brasileiro de Medicina, vol. ıoo, pp. 30-38. 2012.

[3] FERREIRA, G.S. disbiose intestinal: aplicabilidade dos prebióticos e dos probióticos na recuperação e manutenção da microbiota intestinal trabalho de conclusão de curso, Curso de Farmácia, Centro Universitário Luterano De Palmas, 2014.

[4] FERREIRA，R. C.; GONÇALVES，C. M.; MENDES，P. G. Depressão: Do transtorno ao sintoma. Psicologia. O Portal dos Psicólogos, Minas Gerais, p.ı-ı6, i6 nov. 2014.

[5] GONÇALVES, M. A. P. Microbiota - implicações na imunidade e no metabolismo. 20I4. 4If. Dissertação (Mestre em Ciências Farmacêuticas) - Universidade Fernando Pessoa. Porto, 20I4.

[6] Influência da microbiota intestinal e nutrição sobre a depressão em mulheres: uma revisão sistemática.

Brazilian Journal of Health Review, [S.L.], v. 4, n. 2, p. 5622-5638, 2021.

[7] LANDEIRO, J. Impacto da microbiota intestinal na saúde mental. 20I6. 8I $\mathrm{f}$. tese (Mestrado integrado em ciências farmacêuticas) Instituto Superior De Ciências Da Saúde Egas Moniz, Monte de Caparica, Almada, Portugal, 2016.

[8] LEITE, L. et al. Papel da microbiota na manutenção da fisiologia gastrointestinal. Boletim Informativo Geum, v. 5, n. 2, p. 54-6I, 2014.

[9] LOZUPONE, C. A. et al. (2012). Diversity, stability and resilience of the human gut microbiota. Nature, vol. 489, pp. 220-229.

[10] LORENZ, J.; OSORIO, D. R. D. A influência da dieta na composição da microbiota intestinal em obesos: uma revisão. Revista Brasileira de Obesidade, Nutrição e Emagrecimento, São Paulo. v.13. n.83.Suplementar I.p.II59-II67. Jan./Dez.2org.

[11] LAGE, D.G.; BRITO, G.A.P.; a relação da microbiota intestinal com obesidade e resistência à insulina, Revista Brasileira de Obesidade, Nutrição e Emagrecimento, São Paulo v.6, n.31, p.31-34, Jan/Fev.

2012

[12] MUSCOGIURI, G. et al. Microbiota intestinal: um novo caminho para tratar a obesidade. Int J Obes Suppl. v. 9, n. I, p. Io-19, 2019. Disponível em: 
[13] MORAES, A. C. F. et al. Microbiota intestinal e risco cardiometabólico: mecanismos e modulação dietética. Arquivos Brasileiros de Endocrinologia \& Metabologia, v. 58, n. 4, p. 317-327, 2014 .

[14] MUSCOGIURI, G. et al. Microbiota intestinal: um novo caminho para tratar a obesidade. Int J Obes Suppl. v. 9, n. I, p. IO-I9, 2019. Disponível em: https://www.ncbi.nlm.nih.gov/pmc/articles/PMC66831 32. Acesso em: o9 nov. 2020.

[15] MARQUES, I. B. Evidências da relação entre a colonização intestinal por Clostridium difficile e Transtorno do Espectro Autista (TEA). 2016. I8f. Monografia (Graduação) - Faculdade de Ciências da Educação e Saúde, Centro

Universitário de Brasília, Brasília, 2016.

[16] NUNES, M. L.; GARRIDO, M. P. A obesidade e a ação dos prebióticos, probióticos e simbióticos na microbiota intestinal. Nutrição Brasil, 2018; 17(3):189-196.

[17] SANTOS, S. H.; FURTADO, C. C.; PERES, A. L. N. Pré e probióticos no processo de emagrecimento - revisão sistemática. UNILUS Ensino e Pesquisa, v. I6, n. 45, out. / dez. 2019.

[18] OLIVEIRA, A. M.; HAMMES, T. O. Microbiota e barreira intestinal: implicações para obesidade. Clinical \& Biomedical Research, [S.L.], v. 36, n. 4, p. 222-229, 2016.

[19] PARIZOTTO, J.; ZORZ, N. T. Aleitamento materno: fatores que levam ao desmame precoce no município de Passo Fundo, RS. O Mundo da Saúde, São Paulo, v. 32, n. 4, p. 466-474, 2008.

[20] SOARES, D. K. N. S. Modulação da microbiota intestinal com probióticos e sua relação com a obesidade. Revista de Divulgação Científica Sena Aires, [S.L.], p. 356-366, io jul. 2019. Revista de Divulgação Cientifica Sena Aires.

[21] SALOMÃO, J. O. et al. Implicações da microbiota intestinal humana no processo de obesidade e

[22] Emagrecimento: revisão sistemática. Braz. J. Hea. Rev., Curitiba, v. 3, n. 5, p. 15215-15229 set. /out. 2020.

[23] STÜRMER, E.S. et al. A importância dos probióticos na microbiota intestinal Humana, Revista brasileira de Nutrição Clínica, V.27, n.4, nov./fev.2012.

[24] WANDERLEY, E. N.; FERREIRA, V. A. Obesidade: uma perspectiva plural. Ciência \& Saúde Coletiva, v.15, p. 185-194, 2010. 